\title{
Mortalidade de mulheres em idade fértil em Campinas, São Paulo (1985-1994)
}

\author{
Mortality among child bearing-age women \\ in Campinas, São Paulo (1985-1994)
}

Anibal Faúndes 1,2

Mary Angela Parpinelli 2

José Guilherme Cecatti 2

\footnotetext{
1 Centro de Pesquisa das Doenças Materno-Infantis de Campinas, Universidade Estadual de Campinas. C. P. 6181 , Campinas, SP 13081-970, Brasil.

2 Departamento de Tocoginecologia, Faculdade de Ciências Médicas, Universidade Estadual de Campinas.

C. P. 6181 , Campinas, SP 13081-970, Brasil.
}

Abstract To providea profile of the main health problems in childbearing-age women, westudied all 3,086 death certificates from the SEADE Foundation for women from 10 to 49 years of age and residing in the municipality of Campinas, from January 1, 1985, to December 31, 1994. The primary cause of death was identified and classified according to the 10th review of the ICD. Population data were obtained from the Laboratory for Epidemiological Analyses and Research, UNICAMP. One-fourth of the deaths were cardiovascular in origin, one-fifth were from external causes, and al most $20 \%$ were due to neopl asms. Maternal mortality was the ninth cause of death. External causes predominated in the 10-to-34-year age group, as compared to cardiovascular diseases and neoplasms in the 35-to-49-year group. Most alarming were the predominance of traffic accidents among causes of death in women up to age 34 (greater than AIDS during the study period) and the high mortality rate from homicides.

Key words Mortality; FertileAge; Women; Cause of Death

Resumo Com o fim de obter um perfil das principais doenças que afetam as mulheres em idade fértil, estudaram-se todas as 3.086 decl arações de óbito de mulheres de 10 a 49 anos, resi dentes no Município de Campinas, Estado de São Paulo. Óbitos ocorridos entre primei ro de janei ro de 1985 e 31 de dezembro de 1994, fornecidos pela Fundação SEADE. A causa bási ca desencadeante do óbi to foi identificada e classificada segundo a CID 10a revisão. Os dados populacionais para o períodos foram obti dos do Laboratóri o de Análi ses e Pesquisas Epi demi ológi cas da UNICAMP. Um quarto dos óbitos foram por doenças cardi ovasculares e um quinto por causas externas. A terceira causa, próxima a $20 \%$ do total, foi neoplasia. A mortali dade materna constituiu a nona causa de morte. As cau sas externas foram as principais cau sas de morte entre 10 e 34 anos. A partir dos 35 anos, predomi naram as causas cardiovasculares e neoplasi as. Chama a atenção o predomínio dos acidentes de trânsi to como primeira causa de morte ate os 34 anos de idade, superando a AIDS durante o período estudado, assim como a alta mortalidade por homicídio. Palavras-chave Mortalidade; IdadeFértil; Mulheres; Causa da Morte 
Introdução

Nas sociedades econômico, cultural e socialmente mais desenvolvidas, a mortalidade masculina é maior do que a feminina em todas as faixas etárias. Esta condição resulta em uma maior esperança de vida ao nascimento de cerca de sete a oito anos para as mulheres (Banco Mundial, 1990). As análises mais recentes sobre a mortalidade de adultos em países de Primeiro Mundo têm mostrado uma tendência de aumento na mortalidade de mulheres relacionada, principalmente, às mudanças de comportamento e aos hábitos, como: fumar, alimentação inadequada, uso de drogas, sexo desprotegido, maior participação no mercado de trabalho, com predominância de doenças relacionadas a essas atividades (McGinnis \& Foege, 1993; Waldron, 1993).

No Brasil, embora seja um país menos desenvolvido, dados do Banco Mundial (1990) apontam para uma esperança de vida ao nascimento, maior em torno de cinco anos para as mulheres (63 anos para os homens e 68 anos para as mulheres). Esta diferença, à semelhança de países desenvolvidos, vem apresentando tendência à diminuição, como verificado no período de 1980 a 1988, com ganho maior para os homens (seis anos) do que para as mulheres (4,6 anos), tendendo assim à diminuição da distância entre as respectivas esperanças de vida ao nascimento (Chor et al., 1992).

Não existe um coeficiente específico para medir a mortalidade de mulheres em idade reprodutiva, e nem tampouco o intervalo de idade é nítido para a definição deste conceito. Biologicamente é representado pelo período compreendido da menarca à menopausa, mas é amplamente conhecido que a idade da menarca sofre alterações, inclusive secundárias a fatores exógenos, podendo acontecer fisiologicamente dos 10 aos 16 anos. Entretanto, não se pode deixar de considerar a gravidez na adolescência, mesmo em meninas menores de 15 anos. Siqueira \& Tanaka (1986), ao avaliarem a mortalidade entre adolescentes no Brasil, registraram 0,59\% de óbitos secundários à gravidez, parto e puerpério, em meninas de 10 a 14 anos. Dessa forma, recomenda-se que os estudos sobre mortalidade de mul heres em idade reprodutiva devam incluir a faixa etária de 10 a 14 anos, e não somente a partir dos 15 anos (Laurenti, 1988). Apesar de que a idade da menopausa pode sofrer grandes variações, há quase um consenso na idade de 49 anos para definir o término da idade reprodutiva.

Este trabalho propõe-se analisar a mortalidade de mulheres em idade reprodutiva, resi- dentes no Município de Campinas, entre 1985 e 1994, com base em análise pormenorizada das declarações de óbito (DO).

A cidade de Campinas, situada na região sudeste do estado, é um centro industrial, comercial, cultural e médico bastante desenvolvido do Estado de São Paulo, contando ainda com duas universidades. Uma pesquisa sobre as condições de vida (PCV) na cidade de Campinas mostrou que em 1994, 20\% das famílias eram chefiadas por mulheres e um quarto dos chefes era migrante. A renda familiar per capita era de $\mathrm{R} \$ 348,00$, metade das famílias dispunha de até $R \$ 186,00$ de renda familiar per capita, e mais de $36 \%$ tinham renda superior a dez salários mínimos. Vinte porcento das moradias localizavam-se em ruas sem pavimentação, $17 \%$ das famílias não tinham acesso ao sistema público de esgoto, coexistindo situações de excelente nível sócio-econômico e cultural, com um grupo razoável de famílias em situação de extrema pobreza (SEADE, 1995). Estas características especiais da cidade tornam Campinas uma mistura de cidades do terceiro e do primeiro mundo.

Conhecer as cifras de mortalidade, bem como as causas de morte destas mulheres residentes no município, poderá contribuir para o conhecimento dos problemas de saúde que mais freqüentemente acometem este grupo de mulheres, com as possíveis futuras implicações de novas propostas de intervenções para a meIhoria das condições de vida e de saúde desta população.

\section{Casuística e métodos}

Estudaram-se 3.086 DO de mulheres de dez a 49 anos, residentes no Município de Campinas, número que representa o total de óbitos femininos ocorridos no período de primeiro de janeiro de 1985 a 31 de dezembro de 1994, para a faixa etária específica. A fonte primária dos dados de óbitos foi a Fundação SEADE (Fundação Sistema Estadual de Análise de Dados), inicialmente se valendo da listagem de todas as DO, correspondentes a pessoas com as características acima descritas.

De posse da listagem, procedeu-se o levantamento manual das DO, seguido de fotocópias de todas as selecionadas. A etapa seguinte foi a de identificação da causa básica desencadeante do óbito, realizada manualmente por um dos autores (M. A. P.), baseando-se nas informações das DO, segundo a CID 10ạ revisão (OMS, 1995). Contudo, para alguns meses do ano de 1992, foram utilizados os dados tabula- 
dos, segundo a CID 9 com ajuste de equivalência para CID 10, uma vez que houve extravio no arquivo da Fundação SEADE.

Os dados populacionais referentes ao período entre 1985 a 1994 foram obtidos nas estimativas de população residente do IX eX Censo Demográfico do Brasil (1980 e 1991), calculadas pelo Laboratório de Análises e Pesquisas Epidemiológicas, Departamento de Medicina Preventiva e Social, Universidade Estadual de Campinas (LAPE/DMPS/UNICAMP). Para o cálculo foram utilizados dados censitários de 1980 e 1991, aplicando-se progressão geométrica com interpelação e extrapolação. Margem de erro estimada de $4 \%$.

A causa básica do óbito foi definida como a doença ou lesão que iniciou a sucessão de eventos mórbidos que levou diretamente à morte, ou as circunstâncias do acidente ou violência que produziu a lesão fatal (OMS, 1995). Utilizou-se a classificação da causa básica do óbito, segundo os 21 capítulos da CID 10 (OMS, 1995) e segundo a lista de tabulação da mortalidade número 2 (mortalidade geral lista selecionada) - CID - 10.

Pelo preenchimento do formulário específico do estudo, com os ajustes da causa básica, quando necessários, os dados foram revisados, corrigidos manualmente e introduzidos em programa Epi-Info 6.0, para realizar o cálculo dos coeficientes de mortalidade geral e de mortalidade específica por causa ou grupo de causas e por grupo de idade.

Garantiu-se o sigilo das fontes de informação, sem identificação dos sujeitos, profissionais e hospitais que atenderam ou preencheram as DO.

\section{Resultados}

Mortalidade por causas segundo capítulos do CID 10

A principal causa de morte foi o grupo de doenças cardiovasculares, representando um quarto do total da mortalidade, seguido pelas causas externas e neoplasias. A mortalidade materna foi a nona causa de óbito feminino em idade reprodutiva para o período, muito próxima da oitava causa que correspondeu às doenças do aparelho gênito-urinário (Tabela 1). A análise detalhada da mortalidade materna será objeto de uma publicação específica e separada.

A grande maioria dos óbitos aconteceu na cidade de Campinas (96\%) e em ambiente hospitalar (85,1\%). Cerca de $10 \%$ dos óbitos ocorreram no domicílio e $16,3 \%$ das DO foram
Tabela 1

Distribuição percentual e coeficiente de mortalidade por faixa etária de mulheres de 10 a 49 anos, residentes em Campinas de 1985 a 1994, segundo capítulos da CID 10.

\begin{tabular}{lrrc}
\hline Capítulo & $\mathrm{n}$ & $\%$ & Coeficiente $^{1}$ \\
\hline IX (aparelho circulatório) & 781 & 25,3 & 28,6 \\
XX (causas externas) & 610 & 19,8 & 22,3 \\
II (neoplasias) & 605 & 19,6 & 22,1 \\
I (infecto-parasitárias) & 253 & 8,2 & 9,2 \\
X (aparelho respiratório) & 243 & 7,9 & 8,9 \\
XI (aparelho digestivo) & 131 & 4,2 & 4,8 \\
XVIII (mal definidas) & 124 & 4,0 & 4,5 \\
XIV (aparelho gênito-urinário) & 83 & 2,7 & 3,0 \\
XV (gravidez, parto e puerpério)2 & 82 & 2,7 & 3,0 \\
IV (doenças endócrinas) & 66 & 2,1 & 2,4 \\
XIII (doenças do tec. conjuntivo) & 49 & 1,6 & 1,8 \\
VI (doenças do sistema nervoso) & 37 & 1,2 & 1,3 \\
III (doenças hematológicas) & 11 & 0,4 & 0,4 \\
XVII (malformações congênitas) & 9 & 0,3 & 0,3 \\
V (transtornos mentais) & 2 & 0,1 & 0,07 \\
Total & 3.086 & 100 & \\
& & &
\end{tabular}

1 por 100.000 mulheres.

2 Razão de Morte Materna $=44,6 / 100.000 \mathrm{NV}$.

Total de NV de 1985-94 = 183.824.

emitidas pelo Serviço de Verificação de Óbitos (SVO), o que significa que nestes casos ou o óbito ocorreu na residência, ou não houve assistência médica no período da doença.

Mortalidade por idade e capítulo do CID 10

A mortalidade proporcional, bem como seus coeficientes, foi maior à medida que aumenta a idade, sendo o coeficiente mais de dez vezes superior no grupo etário de 45-49 anos que no grupo de 10-14 anos. Onze porcento das muIheres que foram a óbito eram adolescentes e perto de $20 \%$ tinham até 24 anos (Tabela 2 ).

O coeficiente de mortalidade por causas externas manteve-se estável com incidência superior a 20 óbitos/ 100.000 mulheres desde os 20 até os 49 anos de idade e foi a primeira causa de óbito para todos os grupos etários mais jovens, até 30-34 anos, com pico máximo para o grupo etário de 15-19 anos, em que esteve próximo a 30 óbitos/ 100.000 mulheres (Figura 1). As causas cardiovasculares e neoplásicas aumentaram dramaticamente com a idade, passando a ser a primeira e a segunda causa de mortalidade a partir dos 35 anos de idade. 0 maior coeficiente correspondeu às doenças cardiovasculares, com aproximadamente 130 
Tabela 2

Distribuição percentual e coeficiente de mortalidade de mulheres de 10 a 49 anos, residentes em Campinas, de 1985 a 1994, segundo grupo etário.

\begin{tabular}{lrrrr}
\hline Grupo etário & $\begin{array}{l}\text { População } \\
\text { feminina1 }\end{array}$ & n óbitos & $\%$ & Coeficiente $^{2}$ \\
\hline $10-14$ & 400.449 & 128 & 4,1 & 31,9 \\
$15-19$ & 377.192 & 212 & 6,9 & 56,2 \\
$20-24$ & 398.008 & 254 & 8,2 & 63,8 \\
$25-29$ & 405.484 & 338 & 10,9 & 83,4 \\
$30-34$ & 373.710 & 392 & 12,7 & 104,9 \\
$35-39$ & 319.810 & 466 & 15,1 & 145,7 \\
$40-44$ & 256.728 & 571 & 18,5 & 222,4 \\
$45-49$ & 199.075 & 725 & 23,5 & 364,2 \\
Total & 2.730 .456 & 3.086 & 100 & 113,0 \\
\hline
\end{tabular}

1 Somatória da população feminina de dez a 49 anos, estimada segundo faixa etária, para os dez anos (1985 a 1994).

2 por 100.000 mulheres. a idade é menos acentuado para o câncer de colo uterino, e inicia-se aos 25 anos. Para AIDS, os maiores coeficientes estiveram nos grupos etários de 25-29 anos e 40-44 anos.

\section{Discussão}

A validade da DO como instrumento de análise, embora apresentando algumas deficiências na determinação da causa básica, já foi testada previamente e considerada satisfatória, uma vez que o preenchimento é sistematizado e o número de omissões é insignificante para o Estado de São Paulo (Maia \& Campanário, 1994).

O coeficiente de mortalidade de 113,0 por 100.000 mulheres de 10 a 49 anos, encontrado em nosso estudo, e os crescentes coeficientes segundo a idade, de 31,9/100.000 mulheres no grupo etário de 10-14 anos, até 364,2/ 100.000 mulheres na idade de 45-49 anos, são muito semelhantes aos encontrados por Laurenti et al. (1990), no Município de São Paulo, no ano de 1986. Estes autores identificaram um coeficiente de mortalidade de 114,9/ 100.000 mulheres e coeficientes por idade de 30,5/ 100.000 mulheres no grupo etário de dez a 14 anos e 373,0/ 100.000 mulheres de 45-49 anos. Para Carvalheiro \& Manço (1992), no Município de Ribeirão Preto, o coeficiente de mortalidade para a população de mulheres de 15 a 49 anos, no período de 1985 a 1989 foi de 125,1/ 100.000 mulheres, mas estas autoras não avaliaram a faixa etária de 10 a 14 anos. Entretanto, seus coeficientes de mortalidade de 38,4/ 100.000 em mulheres de 15 a 19 anos foram consideravelmente menores que aqueles por nós encontrados, de 56,2/ 100.000 em mulheres na mesma idade. Por outro lado, no grupo etário de 35 anos, nossos coeficientes de mortalidade foram sempre menores, o que sugere diferenças regionais dentro do próprio Estado de São Paulo.

A pequena proporção de óbitos que ocorreu fora do município (4\%) e a alta porcentagem que ocorreu em hospital (85\%) seguramente refletem a capacidade hospitalar de Campinas, e a pequena procura de atenção médica em outras cidades pela população do município. Chama a atenção o número relativamente alto de óbitos cujas DO foram emitidas pelo SVO (16,3\%), que representam casos de morte sem assistência médica ou com atendimento precário que impossibilitou o diagnóstico da causa básica, o que pode ser considerado como indicador de acesso e da qualidade da assistência médica recebida pela população.

A elevada proporção de óbitos por doenças cardiovasculares, neste grupo de mulheres, 
Figura 1

Coeficiente de mortalidade de mulheres de 10 a 49 anos no período de 1985 a 1994, segundo capítulos da CID 10 por faixa etária.

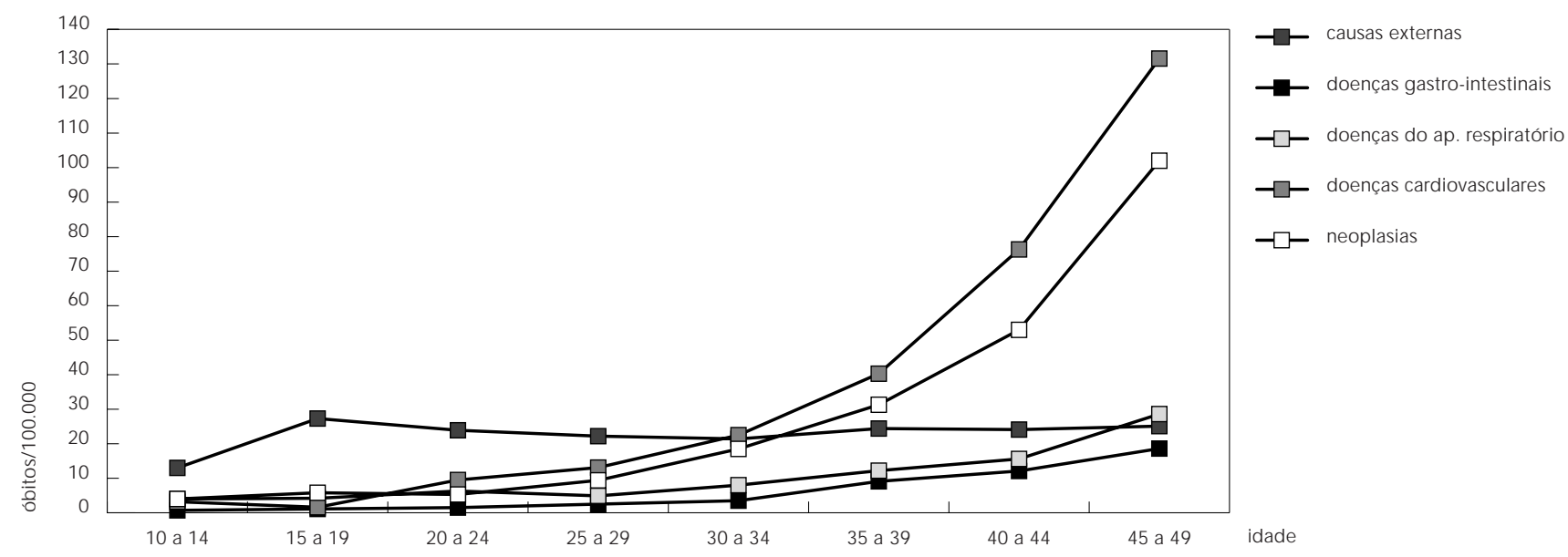

Figura 2

Coeficiente de mortalidade de mulheres de 10 a 49 anos, residentes em Campinas no período de 1985 a 1994 , segundo causas selecionadas e por faixa etária.

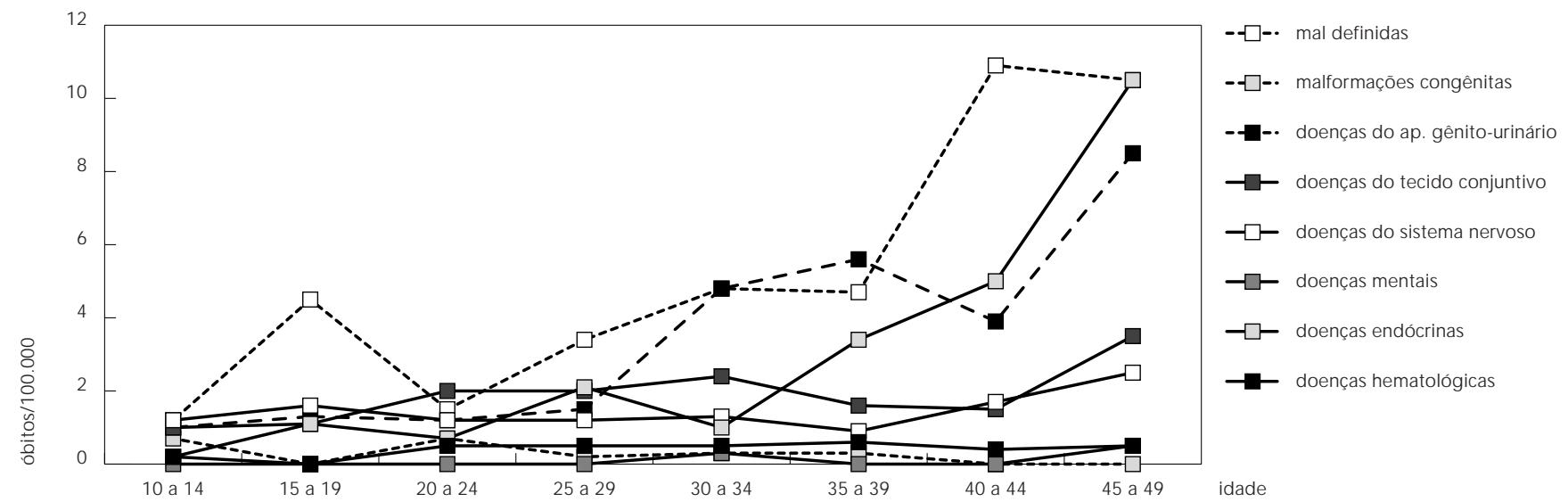

com predomínio crescente a partir do subgrupo de idade 30-34 anos, coincide com os padrões de mortalidade de mulheres em idade reprodutiva avaliados por Laurenti et al. (1990), Lolio et al. (1991) e Carvalheiro \& M anço (1992) em outros municípios do Estado de São Paulo. Esta característica é diferente da observada por Paneque (1986) que, em Cuba, relatou que as doenças cardiovasculares deixaram de ser a pri- meira causa de morte feminina, no grupo de 15 a 49 anos, desde 1968.

Neste contexto, vale ressaltar que, nas últimas décadas, as mortes por doenças cardiovasculares, neoplasias, acidentes e violência, denominadas doenças da industrialização, têm dominado o perfil da mortalidade de adultos nos países desenvolvidos. As doenças cardiovasculares, entretanto, têm decrescido em al- 
Figura 3

Coeficiente de mortalidade de mulheres de 10-49 anos, residentes em Campinas no período de 1985 a 1994, segundo algumas causas e por faixa etária.

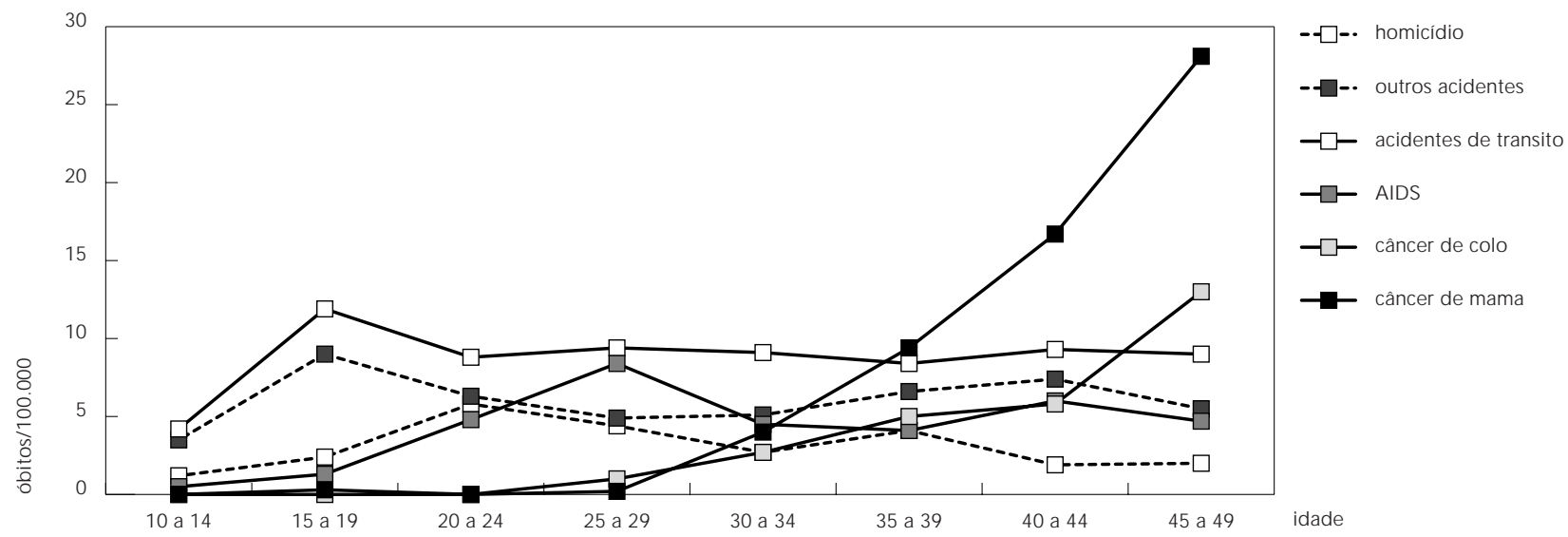

guns grandes centros urbanos, às custas de meIhorias nas condições de diagnóstico e terapêutica especializada, em faixas etárias mais jovens, e passaram a representar parcela importante dos óbitos na população de 65 anos ou mais (Sutherland et al., 1990).

Diversos aspectos podem ser imputados ao predomínio da mortalidade por doenças cardiovasculares na população feminina em idade fértil. No Estado de São Paulo, Lolio et al. (1991), Lotufo \& Lolio (1993a), Lotufo \& Lolio (1993b) discutem a alta prevalência de hipertensão arterial sem diagnóstico no Estado e, portanto, sem tratamento adequado. Outro aspecto relevante a esta situação diz respeito diretamente aos programas de saúde pública aplicados à ginecologia e obstetrícia. Beral (1979) sugere avaliar os óbitos femininos secundários não apenas às complicações do ciclo grávido puerperal, mas também aos que poderiam estar associados à anticoncepção. Neste aspecto, seria importante avaliar a correlação da mortalidade por doenças cardiovasculares pelo uso, por exemplo, de anticoncepcionais orais, em muIheres com hipertensão arterial prévia e/ou com hipertensão secundária ao uso da medicação, ou ao hábito do tabagismo ou com antecedente de fenômenos tromboembólicos. Infelizmente estes aspectos não puderam ser avaliados e, dessa forma, não é possível aprofundar esta discussão, que necessita estudos epidemiológicos específicos para sua melhor elucidação.
Diferente do encontrado por outros autores brasileiros (Lolio et al., 1991; Carvalheiro \& Manço, 1992), para o Estado de São Paulo, em que as causas externas representaram a terceira causa de morte, no grupo estudado estas representaram um quinto da mortalidade no período e foram a segunda causa de óbito, apesar de muito próxima às mortes por neoplasias.

O crescimento da mortalidade secundária às causas externas tem sido avaliado no Brasil, em distintos segmentos da população. VermeIho \& Mello-Jorge (1996) descrevem a evolução da mortalidade de jovens de 15 a 24 anos, nos Municípios de São Paulo e Rio de Janeiro, ocorrida de 1930 a 1991, e revelaram taxas crescentes de mortalidade por causas externas, que passaram a ocupar o primeiro lugar desde a década de 60, e com um crescimento nos coeficientes de até três vezes para o ano de 1991 em relação ao ano de 1960 . Os autores definem esta situação como a transição epidemiológica para a violência.

Esta situação não é peculiar do Brasil. A mortalidade por causas externas tem sido objeto de preocupação pela sua repercussão negativa sobre a qualidade e a expectativa de vida ao nascimento e a estabilidade econômica nas Américas, particularmente desde o ano de 1993, quando a Organização Panamericana de Saúde (OPS) a elegeu como tema da 111a Reunião do Comitê Executivo.

$\mathrm{Na}$ análise da mortalidade por causas externas é importante selecionar seus componen- 
tes, segundo critérios da CID. Yunes \& Rajs (1994), ao avaliarem a tendência desta mortalidade na população das Américas, no período de 1979 a 1990, identificaram comportamento distinto entre os países, em relação aos três grupos principais de mortes por causas externas, acidentes de tráfego, suicídios e violência. Concluem que, embora tenha havido uma tendência de queda na mortalidade por causas externas em nove dos quinze países avaliados, esta dependeu primordialmente da redução dos acidentes de tráfego.

Nossos dados, coincidindo com o verificado por Yunes \& Rajs (1994) para o Brasil, apresentam dentre as causas externas, dois componentes mais alarmantes: os acidentes de trânsito e os homicídios. O coeficiente de mortalidade por acidentes de trânsito, no sexo feminino, encontrado neste trabal ho não é diferente do descrito no Brasil, que, em 1986, foi de 9,4/ 100.000 mulheres, representando o quinto maior do mundo (OMS, 1990/ 1992).

Dados semelhantes foram publicados por Mello-Jorge \& Latorre (1994) que encontraram incremento nos óbitos por acidentes de trânsito no sexo feminino de 7,8 em 1979 para 8,5 em 1983. Nos nossos dados, os acidentes de trânsito constituíram a principal causa de morte de mulheres até o grupo de 30-34 anos, superando inclusive as mortes por AIDS, o que contrasta com a mínima importância que se dá a este problema dentro do setor de saúde.

Assim mesmo, algo semel hante acontece com as questões relacionadas aos homicídios, que no Brasil têm revelado um panorama extremamente negativo e preocupante. Na população estudada, os homicídios apresentaram duas inflexões, uma na faixa etária de 20-24 anos e uma segunda aos 35-39 anos, com coeficientes de mortalidade próximos a 5/ 100.000 mulheres e $3 / 100.000$ respectivamente, muito próximos das taxas para o Brasil de 5,03 no grupo de 20-29 anos encontradas por Souza (1994).

O contexto social e econômico do país nas últimas décadas parece ter papel decisivo nesta situação. As desigual dades sociais e o mercado recessivo de trabalho formal têm propiciado, principalmente aos menos favorecidos, um mercado paralelo, como por exemplo o narcotráfico e o narcoterrorismo.

O jovem é, sem dúvida, mais suscetível e dessa forma tem-se definido um perfil para a violência, caracterizado como indivíduo jovem, pobre e primordialmente do sexo masculino. Os dados deste estudo mostram, entretanto, que o homicídio é também causa importante de morte entre as mulheres na faixa etária avaliada, superando também os óbitos por AIDS nos grupos de até 20-24 anos, no período compreendido por esta pesquisa.

Apesar de as doenças neoplásicas terem ocupado o terceiro lugar entre as causas de morte, neste estudo, estas representaram praticamente um quinto da mortalidade total de mulheres (19,6\% dos óbitos), ficando muito próxima da segunda causa (externas). Este resultado é semelhante aos de outros estudos de mortal idade em idade reprodutiva, no Estado de São Paulo, especificamente nos Municípios de São Paulo e Ribeirão Preto, em que as neoplasias representaram a segunda causa de morte (Laurenti et al., 1990; Carvalheiro \& Manço, 1992). Surpreendente foi o caso do Município de Recife, onde as neoplasias foram a primeira causa de morte, com $28,8 \%$ da mortalidade total de mulheres em idade fértil, nos anos de 1992 e 1993 (Albuquerque et al., 1998).

Vale chamar a atenção que o câncer de mama passou a ser a principal causa de morte por neoplasia, com coeficiente duas vezes maior que para câncer de colo uterino, a partir dos 40 anos de idade, seguindo um padrão mais característico de países desenvolvidos.

A mortalidade por neoplasia de colo uterino, na faixa etária até 44 anos, com coeficientes próximos a 5/ 100.000 habitantes, foi menor inclusive que o de muitos países de primeiro mundo como Inglaterra, em dados do ano de 1992 e Estados Unidos em 1991 (OMS, 1993/ 1994). Por outro lado, o coeficiente de mortalidade para neoplasia cervical ajustado por idade, de aproximadamente 3/ 100.000 mulheres de 10 a 49 anos, neste estudo contrastam com a observação de Albuquerque et al. De acordo com seus dados, é possível estimar um coeficiente de 8,4/ 100.000 mulheres, em idade reprodutiva, no Município de Recife em 19921993 (Albuquerque et al., 1998). A menor mortalidade por esta causa em Campinas talvez possa ser atribuída a um programa de prevenção desta doença, mais antigo e extenso que em outras regiões do país.

O quarto lugar, ocupado pelas doenças infecciosas e parasitárias, com $8,2 \%$ do total de óbitos e coeficiente de mortalidade de 9,2/ 100.000 mulheres no período, coincide com os dados de mortalidade de adultos de 15 a 49 anos em Cuba (Paneque, 1986); com a mortalidade de mulheres em idade reprodutiva, na $\mathrm{Ci}$ dade de São Paulo, no ano de 1986 (Laurenti et al., 1990), e com dados da cidade de Recife, na população de mulheres de 10 a 49 anos, no período de 1992 e 1993 (Albuquerque et al., 1998). Esta semel hança em ambientes que têm diferenças sócio-econômicas evidentes, como no caso de Recife, talvez se explique pela grande 
participação da AIDS neste capítulo. Apesar de a AIDS também se associar ao nível sócio-econômico, sua incidência sofre influência de outras variáveis independe do nível sócio-econômico.

A mortalidade materna representou o nono grupo de causas de morte nesta população, com 2,7\% do total de óbitos, coincidindo com Carvalheiro \& Manço (1992). Foi a quinta causa de morte, com 5,9\% do total de óbitos, na avaliação de Laurenti et al. (1990); a nona, correspondendo a 2,7\% da mortalidade, na avaliação de Carvalheiro \& Manço (1992) e a sétima causa, com $4,1 \%$ do total de mortes de mulheres em idade fértil no estudo de Albuquerque et al. (1998).

De todos os dados obtidos, não há dúvidas de que o que mais chama a atenção de qualquer observador é o lugar destacado que ocupam a violência no trânsito e o homicídio, como causas de morte de mulheres em idade fértil. Enquanto a AIDS e as mortes maternas são objeto de maior destaque no âmbito nacional e mundial (e no caso da AIDS, de amplas e custosas campanhas para sua prevenção e controle), a violência do trânsito e a violência pessoal, apesar de causar um número bem maior de óbitos, não conseguem chamar a atenção, nem de autoridades de saúde, nem de meios de comunicação social, nem da população em geral.

As organizações preocupadas com a saúde da mulher, por exemplo, parecem ainda não ter tomado consciência da importância das causas externas, além da violência doméstica. Os próprios médicos, em posições de influir nas políticas de saúde e educação, ainda não consideraram a gravidade destes problemas.

Se a publicação destes dados conseguir chamar a atenção de colegas, autoridades de saúde e do público em geral, a fim de, sem sensacionalismos, criar consciência para estes problemas, é possível que medidas mais eficientes sejam tomadas para evitar este enorme número de mortes que não deveria acontecer.

\section{Referências}

ALBU QUERQUE, R. M.; CECATTI, J. G.; HARDY, E. E. \& FAÚNDES, A., 1998. Causas e fatores associados à mortalidade de mulheres em idade reprodutiva em Recife, Brasil. Cadernos de Saúde Pública, 14(Sup. 1):41-48.

BANCO MUNDIAL, 1990. Relatório Sobre o Desenvolvimento Mundial 1990: A Pobreza. Rio de Janeiro: Fundação Getúlio Vargas.

BERAL, V., 1979. Reproductive mortality. BMJ, 2:632634.

CARVALHEIRO, C. D. G. \& MANÇO, A. R. X., 1992. Mortalidade feminina no período reprodutivo em localidade urbana da região sudeste do Brasil. Evolução nos últimos 20 anos. Revista de Saúde Pública, 26:239-245.

CHOR, D.; DUCHIADE, M. P. \& JOURDAM, A. M. F., 1992. Diferencial de mortalidade em homens e mulheres em localidade da região sudeste, Brasil 1960, 1970 e 1980. Revista de Saúde Pública, 26: 246-255.

LAURENTI, R., 1988. Marcos referenciais para estudos e investigações em mortalidade materna. Revista deSaúde Pública, 22:507-511. 
LAURENTI, R.; BUCHALLA, C. M.; LOLIO, C. A.; SANTO, A. H. \&JORGE, M. H. P. M., 1990. Mortalidade de mulheres em idade fértil no Município de São Paulo (Brasil), 1986. I. Metodologia e resultados gerais. Revista de Saúde Pública, 24:128-133.

LOLIO, C. A.; LAURENTI, R.; BUCHALLA, C. M.; SANTO, A. H. \&JORGE, M. H. P. M., 1991. Mortalidade de mulheres em idade fértil no Município de São Paulo (Brasil), 1986. III. Mortes por diferentes causas: Doenças cardiovasculares. Revista deSaúdePública, 25:37-40.

LOTUFO, P. A. \& LOLIO, C. A., 1993a. Tendência na mortalidade por doença cerebrovascular no Estado de São Paulo: 1970 a 1989. Arquivos de Neuropsiquiatria, 51:441-446.

LOTUFO, P. A. \& LOLIO, C. A., 1993b. Tendência da mortalidade por doença isquêmica do coração no Estado de São Paulo: 1970 a 1989. Arquivos Brasileiros de Cardiologia, 61:149-153.

MAIA, P. B. \& CAM PANÁRIO, P., 1994. A mortalidade por causas no Estado de São Paulo no período 1980-1992. In: Mortalidadee Sobrevivência no Estado de São Paulo. Informe Demográfico 26. São Paulo: Fundação SEADE.

McGINNIS, J. M. \& FOEGE, W., 1993. Actual causes of death in the United States. JAMA, 270:2207-2212.

MELLO-JORGE, M. H. P. \& LATORRE, M. R. D. O., 1994. Acidentes de trânsito no Brasil: Dados e tendências. Cadernos de Saúde Pública, 10(Sup. 1): 19-44.

OMS (Organização Mundial da Saúde), 1990/ 1992. World Health Statistics Annual, 1989/1991. Genebra: OMS.

OMS (Organização Mundial da Saúde), 1993/ 1994. World Health Statistics Annual, 1992/1993. Genebra: OMS
OMS (Organização Mundial da Saúde), 1995. Classificação Estatística Internacional de Doenças e Problemas Relacionados à Saúde, 10a Revisão, v. 1. São Paulo: Centro Colaborador da OMS para a Classificação de Doenças em Português.

PANEQUE, R. J., 1986. La mortalidad del adulto de 15 a 49 años en Cuba. Niveles y evolución, 19601982. Revista Cubana de Administración de Salud, 12:339-349.

SEADE (Fundação Sistema Estadual de Análise de Dados), 1995. Pesquisa de Condições deVida no Município de Campinas, 1994. Primeiros Resultados. São Paulo: SEADE.

SIQUEIRA, A. A. F. \& TANAKA, A. C. A., 1986. Mortalidade na adolescência com especial referência à mortalidade materna, Brasil, 1980. Revista de Saúde Pública, 20:274-279.

SOUZA, E. R., 1994. Homicídios no Brasil: O grande vilão da saúde pública na década de 80 . Cadernos deSaúde Pública, 10(Sup. 1):45-60.

SUTHERLAND, J. E.; PERSKY, V. W. \& BRODY, J. A., 1990. Proportionate mortality trends: 1950 through 1986. JAMA, 264:3178-3184.

VERM ELHO, L. L. \& MELLO-JORGE, M. H. P., 1996. Mortalidade de jovens: Análise do período de 1930 a 1991 (A transição epidemiológica para a violência). Revista de SaúdePública, 30:319-331.

WALDRON, I., 1993. Recent trends in sex mortality ratios for adults in developed countries. Social Science and Medicine, 36:451-462.

YUNES, J. \& RAJS, D., 1994. Tendencia de la mortalidad por causas violentas en la población general y entre los adolescentes y jóvenes de la región de las Américas. Cadernos de Saúde Pública, 10(Sup. 1):88-125. 